\title{
TUMORES ÓSSEOS BENIGNOS E LESÕES ÓSSEAS PSEUDOTUMORAIS: TRATAMENTO ATUAL E NOVAS TENDÊNCIAS
}

\author{
BENIGN BONE TUMORS AND TUMOR-LIKE BONE LESIONS: \\ TREATMENT UPDATE AND NEW TRENDS
}

José Marcos Nogueira Drumond'

\section{RESUMO}

O tratamento dos tumores ósseos benignos (TOB) e lesões ósseas pseudotumorais (LOP) tem visto surgir novos medicamentos, como os bisfosfonatos de uso intravenoso, que têm mostrado bons resultados no controle das lesões da displasia fibrosa. O cisto ósseo aneurismático tem sido tratado com agentes esclerosantes com sucesso. Tratamentos adjuvantes permitem a realização de cirurgias que preservam a articulação e a função, com baixas taxas de recidiva. Têm sido mais utilizados o cimento ósseo (PMMA), o fenol, a crioterapia com nitrogênio líquido, a água oxigenada, o álcool etílico e a radioterapia. Entre os novos métodos de tratamento surgidos destaca-se a ablação térmica por radiofrequência e por laser, utilizada principalmente para tratamento do osteoma osteoide. A artroscopia permite a ressecção de lesões benignas intra-articulares e assiste na ressecção de tumores subcondrais. Um grande avanço foi a utilização de substitutos sintéticos do osso, que associam substâncias osteoindutivas com material osteocondutivo e têm apresentado resultados comparáveis aos do enxerto ósseo autógeno. Há uma tendência atual para tratamentos fechados, fazendo-se a injeção percutânea de matriz óssea desmineralizada (DBM) associada com sulfato de cálcio. O enxerto ósseo esponjoso autógeno permanece como o padrão ouro. $\mathrm{O}$ enxerto de fíbula vascularizado apresenta os melhores resultados para incorporação em lesões maiores e agressivas. Também o suporte cortical alogênico provê resistência estrutural aumentada nessas lesões mais agressivas. $\mathrm{O}$ aloenxerto liofilizado tem indicação para preencher defeitos contidos e para reforço do enxerto autógeno. As endopróteses articulares são utilizadas em grandes lesões destrutivas no fêmur distal, no quadril e no ombro.

Descritores - Doenças ósseas; Neoplasias ósseas/cirurgia; Neoplasias ósseas/terapia; Neoplasias ósseas/quimioterapia; Neoplasias ósseas/radioterapia

\section{ABSTRACT}

The treatment of benign bone tumors (BBT) and tumor-like bone lesions (TBL) has observed the introduction of new drugs, such as intravenous bisphosphonates, which have ossified bone lesions caused by fibrous dysplasia. Aneurismal bone cyst has been treated with sclerosing agents by percutaneous injection, yielding good results. Adjuvants allow joint salvage, maintenance of movements and function, with low rates of recurrence. Among them, the most used ones are bone cement (PMMA), phenol, nitrogen-based cryotherapy, hydrogen peroxide, ethanol and radiotherapy. New methods of treatment include thermal ablation with radiofrequency and laser, mainly utilized for treating osteoid osteoma. Arthroscopy allows resection of benign intra-joint lesions and assists the surgery of subchondral tumors. A great advance is the utilization of synthetic bone substitutes, which are a mixture of osteoinductive growth factors and osteoconductive ceramics, and have presented comparable results to autogenous bone grafts. There is a recent trend for closed treatments, with percutaneous injection of demineralized bone matrix (DBM) and calcium sulfate. Autogenous cancellous bone graft remains as the gold standard. Vascularized fibula graft, on the other hand, incorporates faster in the treatment of large destructive lesions. Also, allogenic cortical support allows structural augmentation for aggressive tumors. Freeze-dried allografts are used to fill contained defects and as expanders of autografts. Joint endoprosthesis may be used in large destructive lesions of the distal femur, hip and shoulder.

Keywords - Bone diseases; Bone neoplasms/surgery; Bone neoplasms /therapy; Bone neoplasms /chemotherapy; Bone neoplasms/ radiotherapy

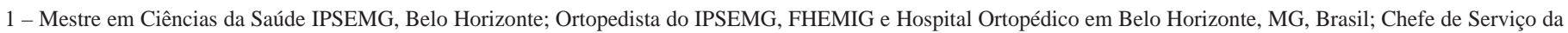
FHEMIG - Fundação Hospitalar do Estado de Minas Gerais.

Trabalho realizado no IPSEMG - Instituto de Previdência dos Servidores do Estado de Minas Gerais, Belo Horizonte, MG, Brasil. Correspondência: Rua Prof. Estevão Pinto, 555 /304, Serra - 30220-060 - Belo Horizonte, MG. E-mail: jmnd@uai.com.br. 


\section{INTRODUÇÃO}

Os tumores ósseos benignos primários são relativamente raros, o que gera certa dificuldade para seu diagnóstico e tratamento. As lesões ósseas pseudotumorais são lesões não neoplásicas que simulam tumores ósseos. É importante o seu conhecimento porque podem ser confundidas com tumores ósseos e receberem tratamento excessivo ou inadequado.

Os tumores ósseos benignos e as lesões ósseas pseudotumorais ocorrem com maior frequência na faixa etária dos cinco aos 25 anos e em localizações de maior crescimento ósseo, com cerca de $60 \%$ dos casos na região do joelho. Por isso, podem provocar deformidades osteoarticulares, fraturas, discrepância de comprimento de membros, distúrbios da marcha, às vezes com sequelas incapacitantes.

O tratamento dessas lesões tem apresentado recentes e contínuas inovações, como a utilização de novos medicamentos, novas modalidades de adjuvantes, enxertia com substitutos ósseos sintéticos e técnicas avançadas de artroscopia e radiofrequência.

O objetivo deste trabalho foi descrever o que é mais atual e as novas tendências no tratamento dos tumores ósseos benignos (TOB) e lesões ósseas pseudotumorais (LOP).

\section{TRATAMENTO}

\section{Medicamentos}

Alguns tumores benignos podem ser tratados com diferentes tipos de medicamentos. O conhecimento e o tratamento da displasia fibrosa evoluíram muito nos últimos dez anos. Devido à sua agora conhecida natureza genética, explicam-se as recidivas frequentes após a curetagem e enxertia óssea, seu tratamento tradicional. Recentemente, a doença vem sendo tratada com bisfosfonato intravenoso, que atua inibindo a reabsorção óssea mediada por osteoclastos. Resultados iniciais na displasia fibrosa têm mostrado diminuição na dor, prevenção de fraturas e resolução radiográfica parcial das lesões. Pamidronato é o bisfosfonato de terceira geração mais utilizado e é administrado mensalmente por infusão intravenosa em período de duas a quatro horas. Já é utilizado, com bons resultados também, na doença de Paget e na doença metastática óssea. O ácido zoledrônico e outros bisfosfonatos têm atividade antitumoral e podem reduzir a carga tumoral óssea, prevenir metástases e inibir a progressão de lesões ósseas ${ }^{(1)}$.

Para o tratamento do cisto ósseo simples, a medicação ainda mais utilizada para infiltração é o acetato de metilprednisolona, com bons resultados ${ }^{(1)}$.
A utilização de agentes fibrosantes para o tratamento do cisto ósseo aneurismático (COA) apresenta resultados controversos. O tratamento com a solução alcoólica de Zein apresentou altas taxas de complicações locais e sistêmicas e tem sido abandonado ${ }^{(2)}$. A escleroterapia com polidocanol mostrou-se mais segura e eficaz, com resultados de longo seguimento publicados ${ }^{(3)}$.

\section{Adjuvantes}

O uso de adjuvantes visa a erradicação da doença microscópica por meios térmicos e químicos. A tendência atual é pela preservação da articulação com cirurgia intralesional e uso de adjuvantes no tratamento das lesões ósseas benignas ${ }^{(4,5)}$. A excisão ampla apresenta menores taxas de recidiva, mas com piores resultados funcionais.

O tratamento do tumor de células gigantes (TCG) com excisão intralesional, cauterização e utilização de fenol mais cimento ósseo (polimetilmetacrilato, PMMA) como adjuvantes tem mostrado excelentes resultados funcionais e baixas taxas de recidiva e de artrose secundária $^{(4)}$.

O cimento ósseo provê estabilidade estrutural imediata, mas ainda não há evidências de haver efeito tumoricida por necrose térmica das células tumorais. O fenol é um agente citotóxico não seletivo, atua sobre outros tecidos e é sistemicamente tóxico. É usado localmente na cavidade e produz morte celular superficial por 1 a $2 \mathrm{~mm}^{(6)}$.

A crioterapia com nitrogênio líquido é tratamento curativo para tumores benignos agressivos e tumores ósseos malignos de baixo grau ${ }^{(7)}$. É também útil no controle local e alívio sintomático em doença óssea metastática. A necrose óssea produzida a partir da cavidade torna a criocirurgia, que é intralesional por definição, tão efetiva quanto uma excisão ampla. A técnica cirúrgica consiste em exposição, curetagem e ampliação da margem com broca rotatória, criocirurgia, reconstrução com cimento ósseo, fixação interna e enxerto ósseo subcondral. A osteossíntese e a proteção contra impactos por seis meses são importantes para evitar uma fratura patológica porque a regeneração óssea ocorre devagar ${ }^{(7)}$.

A criocirurgia pode produzir necrose celular de até 2cm a partir da superfície óssea e nesse aspecto é mais efetiva do que o cimento e o fenol. Para reduzir a taxa de complicações da criocirurgia com instilação direta do nitrogênio líquido em sistema aberto, novas técnicas foram idealizadas para permitir controle de temperatura e tempo de congelamento e para que toda a cavidade, independente da forma geométrica e irregularidades, seja igualmente tratada. Utiliza-se spray pressurizado de nitrogênio líquido e gás argônio ${ }^{(6,8)}$. 
A água oxigenada $\left(\mathrm{H}_{2} \mathrm{O}_{2}\right)$ é usada clinicamente como adjuvante químico para remoção de células tumorais residuais em cirurgia de curetagem intralesional para TCG. Provoca lise e morte das células tumorais e associada ao cimento ósseo tem apresentado baixas taxas de recidiva $^{(9)}$. O álcool etílico tem sido usado no osteoma osteoide através de injeção no nidus guiado por tomografia computadorizada (TC) ${ }^{(5,10)}$.

A radioterapia é bastante efetiva em interromper a progressão ou conseguir resolução completa e permanente para doenças mesenquimais benignas, mas existe pequeno risco de malignização da lesão ${ }^{(11)}$. Apresenta bons resultados no hemangioma de corpo vertebral e do sacro. No TCG e COA pode ser indicada para lesões com difícil acesso ou recidivadas na coluna e pelve ou em pacientes sem condições médicas para cirurgia. Nos tumores primários ou metastáticos do sacro indica-se a radioterapia isolada ou combinada com cirurgia e radiocirurgia ${ }^{(12)}$.

\section{Radiofrequência}

A ablação por radiofrequência utiliza ondas longas de radiação eletromagnética para produzir coagulação térmica.

Estudo comparativo entre ablação percutânea guiada por TC e tratamento cirúrgico aberto para o osteoma osteoide sugere que ambos os métodos são essencialmente equivalentes ${ }^{(13)}$. O método percutâneo por radiofrequência é preferido porque não necessita de hospitalização, não está associado a complicações e permite rápida convalescença. Está indicado para lesões que não estejam intimamente adjacentes a estruturas neurovasculares no esqueleto apendicular e pelve. Está contraindicado na mão e coluna. A biópsia com agulha é realizada no mesmo ato cirúrgico. Duas grandes séries de pacientes tratados no Massachusetts General Hospital (MGH) e Istituti Ortopedici Rizzoli estão publicadas ${ }^{(14,15)}$.

A técnica tem sido usada mais recentemente para o tratamento do condroblastoma ${ }^{(16)}$, da dor em metástases ósseas e de recidivas de cordoma ${ }^{(15)}$.

A terapia térmica com laser guiado por TC também tem sido bem sucedida. A energia do laser transmitida por cabo de fibra óptica produz aquecimento e necrose celular ${ }^{(5,15)}$.

\section{Artroscopia}

Biópsia e tratamento cirúrgico artroscópicos são recomendados para lesões intra-articulares selecionadas, como sinovite vilonodular pigmentada, condromatose sinovial, lipoma arborescente, angioma sinovial, lipoma sinovial intra-articular, osteocondroma ${ }^{(17-19)}$.

Até procedimentos minimamente invasivos como a artroscopia podem ter consequências adversas na pre- sença de neoplasia não suspeitada. Antes da artroscopia devem-se fazer exames não invasivos, incluindo a ressonância magnética quando indicada, para descartar lesão tumoral. Artroscopia indevida pode contaminar a articulação e impedir uma cirurgia de salvamento do membro e até levar à amputação ${ }^{(17)}$.

Adicionalmente, a artroscopia é um auxiliar útil durante o tratamento cirúrgico aberto de pacientes com certos tumores ósseos benignos justarticulares, como o TCG e o condroblastoma. A curetagem artroscópica tem limitações na técnica e na biologia tumoral e não é o procedimento standard até o momento. A curetagem deve ser feita através de janela óssea cortical, com assistência da artroscopia para visualização das superfícies articulares e observar a integridade articular, enquanto prosseguem a curetagem subcondral aberta e o preenchimento com enxerto ou cimento, sem danificar a cartilagem articular ${ }^{(17,20)}$.

O osteoma osteoide justarticular no joelho e no quadril, envolvendo o colo femoral e o acetábulo, pode ser ressecado com o auxílio da artroscopia e TC, com morbidade mínima, excelente alívio dos sintomas e rápida recuperação funcional ${ }^{(20,21)}$.

Artroscopia tem sido realizada para ressecção de exostoses intra e periarticulares no quadril e joelho, provocadoras de dor, limitação de movimentos, subluxação e dano articular precoce. O risco de lesões iatrogênicas e necrose da cabeça femoral é menor porque não há necessidade de luxação do quadril para acessar a exostose ${ }^{(18,19)}$.

Curetagem endoscópica sem enxertia óssea pode ser usada em casos de tumores ósseos benignos extra-articulares sem extensão para partes moles. A cavidade do tumor é suficientemente grande para a endoscopia ${ }^{(22)}$.

\section{Substitutos sintéticos do osso}

O enxerto ósseo sintético ideal deve prover fatores de crescimento osteoindutivos, células mesenquimais primitivas indiferenciadas (células-tronco), material osteocondutivo para criar um arcabouço estrutural, ambiente favorável para boa função das células e formação de osso novo com integração completa ao hospedeiro ${ }^{(23,24)}$.

São osteoindutivos a matriz óssea desmineralizada $(\mathrm{DBM})^{(25)}$, a proteína morfogenética óssea (BMP) e os fatores de crescimento (de fibroblasto, plaquetas, insulina e outros) ${ }^{(23)}$. Injeções de medula óssea autóloga, com células-tronco multipotenciais, promovem a osteogênese.

São osteocondutivos as cerâmicas para reconstrução e a principal é o fosfato de cálcio, incluindo ainda a hidroxiapatita (HA), o fosfato tricálcico (TCP) ou a combinação desses dois (HA-TCP) ${ }^{(26,27)}$, e o sulfato de cálcio (gesso) ${ }^{(23,24,28,29)}$. 
Os enxertos compósitos são formados de materiais que incluem matriz osteocondutiva combinada ou com células osteogênicas ou fatores de crescimento osteoindutivos $^{(23,24,28,29,30)}$. Muito usados em reconstrução craniofacial, tumores ósseos benignos, como expansores de enxerto ósseo ou substitutos de enxerto em fraturas estabilizadas e pseudoartroses. Apresentam resultados comparáveis aos do enxerto ósseo autógeno e sem morbidade. São promessas de ultrapassar o bem estabelecido "padrão-ouro" autógeno ${ }^{(23,30)}$.

Há uma tendência atual para tratamentos fechados, minimamente invasivos, de lesões benignas, como o fibroma não ossificante, cisto ósseo simples e aneurismático, com injeção percutânea, sem curetagem aberta, de matriz óssea desmineralizada (DBM) associada com sulfato de cálcio ${ }^{(30)}$ ou com aspirado de medula óssea de crista ilíaca, que provê células- tronco ${ }^{(1,31)}$. Fosfato de cálcio e hidroxiapatita injetáveis têm sido usados em fraturas e tumores benignos, funcionando como cimento e produzindo resistência mecânica ${ }^{(24)}$.

\section{Enxertos ósseos}

O enxerto ósseo esponjoso autógeno é considerado o padrão-ouro. Tem os componentes básicos para integração e cura: fatores de crescimento osteoindutores, matriz osteocondutora, células-tronco osteogênicas de medula óssea ${ }^{(23)}$. Desvantagens seriam a morbidade cirúrgica na área doadora e poucos sítios doadores no sistema esquelético, com quantidade limitada de enxerto.

Enxertos de fíbula vascularizados são incorporados mais rapidamente que os não vascularizados e apresentam resultados superiores no tratamento dos tumores ósseos benignos agressivos ${ }^{(32)}$.

Cirurgia com transferência de autoenxerto osteocondral pode ser realizada para tratamento de tumores benignos justapostos à cartilagem articular e com erosão da mesma. O enxerto geralmente é removido do côndilo lateral do fêmur ${ }^{(33)}$.

O aloenxerto seria menos desejável pelo risco de rejeição imunológica, potencial para transmissão de doenças, infecções e inferioridade biológica para incorporação. Tem propriedades osteocondutoras, mas potencial osteoindutivo limitado ${ }^{(23)}$. A desmineralização aumenta sua performance biológica e melhor processamento diminui o risco de transmitir infecção. O aloenxerto congelado é mais usado para reconstrução osteoarticular em grandes ressecções e o aloenxerto liofilizado para preencher defeitos contidos e para reforço do enxerto ósseo autógeno ${ }^{(24)}$.
No tratamento de TOB e LOP com grandes defeitos ósseos, a técnica reconstrutiva utilizando suporte cortical alogênico provê resistência aumentada, fácil fixação, remodelação do defeito cístico, consolidação de fraturas e prevenção de deformidade. Porém, a remodelação ocorre devagar e pode não ser completa. Podem ser associados adjuvantes como álcool e fenol, enxerto ósseo alogênico esponjoso e osteossíntese, se necessária (geralmente no fêmur) ${ }^{(34)}$.

\section{Embolização}

A embolização arterial seletiva pode ser utilizada no pré-operatório, como adjuvante, para reduzir o sangramento cirúrgico, ou como tratamento isolado, definitivo, quando a cirurgia é contraindicada ${ }^{(1,31,35)}$.

Embora a cirurgia seja o principal tratamento para muitos tumores sacrais benignos, malignos e metastáticos, a embolização é uma terapia de valor, primária ou adjuvante. Pacientes com lesões benignas, incluindo COA e TCG, respondem à embolização com resolução dos sintomas e ossificação das lesões ${ }^{(36)}$.

\section{Cirurgia}

A ressecção do fêmur distal e reconstrução com endoprótese não convencional é uma técnica segura e confiável que provê boa função e controle local do tumor na maioria dos pacientes. Retalhos de músculo gastrocnêmio são utilizados na reconstrução de partes moles. Está indicada para tumores malignos e benignos agressivos como o TCG e a taxa de salvamento de membros chega a $96 \%{ }^{(37)}$.

As endopróteses são úteis também na reconstrução de grandes defeitos no fêmur e no úmero proximais. Outras técnicas cirúrgicas que podem ser utilizadas para reconstrução articular são os compósitos aloprotéticos e os aloenxertos osteoarticulares maciços associados com osteossíntese $^{(24)}$. A enxertia óssea autógena ou alógena tem indicação também quando da realização de artrodeses.

O tratamento mais utilizado para o cisto ósseo aneurismático e outros tumores benignos, como o condroblastoma, encondroma, osteoblastoma, fibroma condromixoide, é a excisão marginal extracapsular utilizando broca de alta velocidade e o preenchimento da cavidade com enxerto ósseo autógeno ou alógeno. Adjuvantes como o fenol, o nitrogênio líquido e o cimento ósseo podem ser utilizados em lesões agressivas e recidivadas, com redução das taxas de recorrência ${ }^{(1,5,31,35)}$. A excisão ampla apresenta menor risco de recidiva e é mais indicada em ossos dispensáveis e em lesões excêntricas em ossos longos. 


\section{REFERÊNCIAS}

1. Biermann JS. Common benign lesions of bone in children and adolescents. J Pediatr Orthop. 2002;22(2):268-73.

2. Topouchian V, Mazda K, Hamze B, Laredo JD. Aneurysmal bone cysts in children: complications of fibrosing agent injection. Radiology. 2005;236(3):1111.

3. Rastogi S, Varshney MK, Trikha V, Khan SA, Choudhury B, Safaya R. Treatment of aneurysmal bone cysts with percutaneous sclerotherapy using polidocanol: a review of 72 cases with long-term follow-up. J Bone Joint Surg Br. 2006;88(9):1212-6.

4. Saiz P, Virkus W, Piasecki P, Templeton A, Shott S, Gitelis S. Results of giant cell tumor of bone treated with intralesional excision. Clin Orthop Relat Res. 2004;(424):221-6.

5. Gibbs CP, Lewis VO, Peabody T. Beyond bone grafting: techniques in the surgical management of benign bone tumors. Instr Course Lect. 2005;54:497-503.

6. Dabak N, Tomak Y, Piskin A, Gulman B, Ozcan H. Early results of a modified technique of cryosurgery. Int Orthop. 2003;27(4):249-53.

7. Bickels J, Meller I, Shmookler BM, Malawer MM. The role and biology of cryosurgery in the treatment of bone tumors. A review. Acta Orthop Scand. 1999;70(3):308-15.

8. Bickels J, Kollender Y, Merimsky O, Isaakov J, Petyan-Brand R, Meller I. Closed argon-based cryoablation of bone tumours. J Bone Joint Surg Br. 2004 Jul;86(5):714-8.

9. Nicholson NC, Ramp WK, Kneisl JS, Kaysinger KK. Hydrogen peroxide inhibits giant cell tumor and osteoblast metabolism in vitro. Clin Orthop Relat Res. 1998;(347):250-60.

10. Jones KB, DeYoung BR, Morcuende JA, Buckwalter JA. Ethanol as a local adjuvant for giant cell tumor of bone. lowa Orthop J. 2006;26:69-76.

11. Suit $\mathrm{H}$, Spiro I. Radiation treatment of benign mesenchymal disease. Semin Radiat Oncol. 1999;9(2):171-8.

12. Gibbs IC, Chang SD. Radiosurgery and radiotherapy for sacral tumors. Neurosurg Focus. 2003;15(2):E8.

13. Rosenthal DI, Hornicek FJ, Wolfe MW, Jennings LC, Gebhardt MC, Mankin HJ Percutaneous radiofrequency coagulation of osteoid osteoma compared with operative treatment. J Bone Joint Surg Am. 1998;80(6):815-21.

14. Torriani M, Rosenthal DI. Percutaneous radiofrequency treatment of osteoid osteoma. Pediatr Radiol. 2002;32(8):615-8.

15. Rimondi E, Bianchi G, Malaguti MC, Ciminari R, Del Baldo A, Mercuri M, et al. Radiofrequency thermoablation of primary non-spinal osteoid osteoma: optimization of the procedure. Eur Radiol. 2005;15(7):1393-9.

16. Erickson JK, Rosenthal DI, Zaleske DJ, Gebhardt MC, Cates JM. Primary treatment of chondroblastoma with percutaneous radio-frequency heat ablation: report of three cases. Radiology. 2001;221(2):463-8.

17. Bahamonde L, Catalan J. Bone tumors around the knee: risks and benefits of arthroscopic procedures. Arthroscopy. 2006;22(5):558-64.

18. Bonnomet F, Clavert P, Abidine FZ, Gicquet P, Clavert JM, Kempf JF. Hip arthroscopy in hereditary multiple exostoses: a new perspective of treatment Arthroscopy. 2001;17(9):E40.

19. Schomver S, Ciullo JV. Arthroscopic resection of an osteochondroma of the knee. Arthroscopy. 2001;17(7):765-7.
20. Franceschi F, Marinozzi A, Rizzello G, Papalia R, Rojas M, Denaro V. Computed tomography-guided and arthroscopically controlled en bloc retrograde resection of a juxta-articular osteoid osteoma of the tibial plateau. Arthroscopy. 2005;21(3):351-9.

21. Khapchik V, O'Donnell RJ, Glick JM. Arthroscopically assisted excision of osteoid osteoma involving the hip. Arthroscopy. 2001;17(1):56-61.

22. Otsuka T, Kobayashi M, Yonezawa M, Kamiyama F, Matsushita Y, Matsui N. Treatment of chondroblastoma of the calcaneus with a secondary aneurysmal bone cyst using endoscopic curettage without bone grafting. Arthroscopy. 2002;18(4):430-5.

23. Joseph M. Lane, Safdar N. Khan. Bone grafts of the 20th century: multiple purposes, materials and goals. Orthopedics Today. 2000.

24. Rougraff BT. Bone graft alternatives in the treatment of benign bone tumors. Instr Course Lect. 2005;54:505-12.

25. Douglas W. Jackson. Using DBMs in clinical orthopedics. Orthopedics Today. 2005;25:20.

26. Yamamoto T, Onga T, Marui T, Mizuno K. Use of hydroxyapatite to fill cavities after excision of benign bone tumours. Clinical results. J Bone Joint Surg Br. 2000;82(8):1117-20.

27. Matsumine A, Myoui A, Kusuzaki K, Araki N, Seto M, Yoshikawa $H$, et al. Calcium hydroxyapatite ceramic implants in bone tumour surgery. A long-term follow-up study. J Bone Joint Surg Br. 2004;86(5):719-25.

28. Gitelis S, Virkus W, Anderson D, Piasecki P, Yao TK. Functional outcomes of bone graft substitutes for benign bone tumors. Orthopedics. 2004;27(1 Suppl):S141-4.

29. Kelly CM, Wilkins RM, Gitelis S, Hartjen C, Watson JT, Kim PT. The use of a surgical grade calcium sulfate as a bone graft substitute: results of a multicenter trial. Clin Orthop Relat Res. 2001;(382):42-50.

30. Wilkins RM, Kelly CM. The effect of allomatrix injectable putty on the outcome of long bone applic ations. Orthopedics. 2003;26(5 Suppl):S567-70.

31. Cottalorda J, Bourelle S. Modern concepts of primary aneurismal bone cyst. Arch Orthop Trauma Surg. 2007;127(2):105-14.

32. Chen Z, Chen Z, Zhang G. Fibula grafting for treatment of aggressive benign bone tumor and malignant bone tumor of extremities. Chin Med $\mathrm{J}$. 1997;110(2):125-8.

33. Anderson AF, Ramsey JR. Chondroblastoma of the talus treated with osteochondral autograft transfer from the lateral femoral condyle. Foot Ankle Int. 2003;24(3):283-7.

34. Shih HN, Chen YJ, Huang TJ, Hsu KY, Hsu RW. Semistructural allografting in bone defects after curettage. J Surg Oncol. 1998;68(3):159-65

35. Yu GV, Roth LS, Sellers CS. Aneurysmal bone cyst of the fibula. J Foot Ankle Surg. 1998;37(5):426-36

36. Gottfried ON, Schmidt MH, Stevens EA. Embolization of sacral tumors. Neurosurg Focus. 2003;15(2):E4.

37. Bickels J, Wittig JC, Kollender Y, Henshaw RM, Kellar-Graney KL, Meller I, Malawer MM. Distal femur resection with endoprosthetic reconstruction: a longterm followup study. Clin Orthop Relat Res. 2002;(400):225-35. 